\title{
Special Issue on Assistive and Rehabilitation Robotics
}

\author{
Samer Mohammed ${ }^{1}$ - Hae Won Park ${ }^{2}$. Chung Hyuk Park ${ }^{3}$ - Yacine Amirat ${ }^{1}$. \\ Brenna Argall ${ }^{4}$
}

Published online: 18 February 2017

(C) Springer Science+Business Media New York 2017

\section{Introduction}

Assistive and rehabilitation robotics currently covers a broad spectrum of research areas, from intelligent robots that act as aides, companions and educators; to robots that provide physical assistance for mobility, manipulation and support; to embedded robotics, ambient intelligence and intelligent spaces. People whose ability to perform daily living activities is limited or inhibited by injury, disease or impairment include (but are not limited to) stroke survivors with sustained neurological injuries, elderly populations with skeletal muscle weakness, persons with low-vision or blindness, traumatic injuries to the brain or spinal cord, and a variety of degenerative diseases that affect cognition, muscle tone and motor control.

Samer Mohammed

samer.mohammed@u-pec.fr

Hae Won Park

haewon@mit.edu

Chung Hyuk Park

chpark@gwu.edu

Yacine Amirat

amirat@u-pec.fr

Brenna Argall

brenna.argall@northwestern.edu

1 Université Paris-Est Créteil, 120-122, Rue Paul Armangot, 94400 Vitry-sur-Seine, France

2 MIT Media Lab, 20 Ames St, E15-450, Cambridge, MA 02142, USA

3 The George Washington University, Washington, DC 20052, USA

4 Northwestern University and the Rehabilitation Institute of Chicago, Chicago, IL 60611, USA
Over the last decade, robotics technologies increasingly interact closely with the humans they are assisting. Improvements in sensor technologies, hardware design and control have enabled this. Augmenting assistive machines with robotics intelligence in particular has made great stridesincluding intelligence found in wearable robots, prostheses, intelligent walkers and canes, and autonomy found in wheelchairs and robotic arms. Moreover, the emergence of novel adapted technologies, such as those in wearable sensors and soft robots, are gaining in popularity - and not the least because of considerable reductions in cost, size and energy consumption. There also has been a considerable push for assistive robots that serve as cognitive and educational aides, and provide coaching to improve health outcomes. Importantly, subject studies with such robots performed in-situ-in the human's environment rather than the laboratory-have been gaining momentum.

\section{Foundational Workshops}

This Special Issue on Assistive and Rehabilitation Robotics grew out of the joint efforts of three distinct workshops that were organized in conjunction with the 2014 IEEE/RSJ International Conference on Robotics and Intelligent Systems (IROS), held in Chicago, Illinois USA, from September 14th to 18th, 2014. These workshops were entitled: "Assistance and Service Robotics in a Human Environment", "Rehabilitation and Assistive Robotics: Bridging the Gap Between

\footnotetext{
1 Anne Spalanzani, Yacine Amirat, David Daney, Samer Mohammed, Ren Luo, Rachid Alami, and Christian Laugier, IROS 2014 Workshop on "Assistance and Service Robotics in a Human Environment", http:// www.lissi.fr/iros-ar2014/doku.php.
} 
Clinicians and Roboticists", 2 and "Assistive Robots for Individuals with Disabilities: HRI Issues and Beyond". 3

The three workshops gathered leading researchers in the field of assistive and rehabilitation robotics. Fruitful discussion among the speakers and the attendees centered around the state of the art and the challenges and limiting factors for developing sustainable robotics solutions for human assistance and rehabilitation.

Following the three workshops, we guest editors launched this Special Issue through a call for papers that included the participants of the three workshops and also the larger research community. Following the open call, we collected 98 extended abstracts on topics ranging from human-robot interaction and social interaction, to healthcare and well-being, to intelligent habitats and mobility assistance. After a careful review process, 22 abstracts were invited to submit full papers. Of these, 14 papers were accepted for publication in the Special Issue.

\section{Content of the Special Issue}

The papers of the Special Issue cover a broad range of topics, from robots that assist with manipulation and mobility (Sect. 3.1), to those that aid in education, cognitive and physical rehabilitation using social interaction capabilities (Sect. 3.2), to those that physically augment the human body (Sect. 3.3).

\subsection{Assistive Robots for Manipulation and Mobility}

The introduction of robotics autonomy to assistive machines used for mobility and manipulation can make the control of the machine easier, especially when a person has upper-body or cognitive impairments.

For robotic wheelchairs, much work has been done in the area of control assistance and short-distance route planning indoors, however less work has been done in the area of long-distance route planning in outdoor environments. In this Special Issue, the contribution of Schwesinger et al. (2016) does just this. The focus of their work is on robust perception in unstructured, outdoor environments, and map-based localization in these environments-notably without relying on GPS.

\footnotetext{
$\overline{2}$ Brenna Argall and Siddhartha Srinivasa, IROS 2014 Workshop on "Rehabilitation and Assistive Robotics: Bridging the Gap Between Clinicians and Roboticists", http://users.eecs.northwestern. edu/ argall/14rar/.

${ }^{3}$ Hae Won Park, Momotaz Begum, and Chung Hyuk Park, IROS 2014 Workshop on "Assistive Robots for Individuals with Disabilities: HRI Issues and Beyond", http://www.haewonpark.com/ IROS2014-ARHRI/.
}

The field of robotic wheelchair research has moved in recent years away from the idea of full autonomy. However there remains no clear consensus within the literature on which is the right way to accomplish shared control, and there is reason to believe that what is "right" may differ for each person. The work of Viswanathan et al. (2016) contributed to this Special Issue gathers user feedback about various control modes, with a focus on different levels of cognitive impairment in the human operator. In order to first solicit user-feedback before complete system building, they opt for a wizard-of-oz paradigm that has a human experimenter provide the assistive control commands.

For some, the operation of a mobility aide is hampered not (or not only) by their motor impairment but rather by some additional impairment. The Special Issue contribution of Wachaja et al. (2016) targets difficulties faced by persons with low-vision or blindness when operating mobility aides like walkers. They introduce a smart walker that provides both navigation guidance and information about the surrounding environment to the human, and makes use of vibro-tactile interfaces.

In addition to replacing or augmenting lost or diminished function, robotics technologies used for manipulation and mobility also might take the form of physical aides or task partners.

Robotic aides that assist aging populations is a quite active research area. One key concern, and identified area for robotic assistance, are transfers-for example, in and out of a bed or a wheelchair, or from sitting to standing_-because of the risk of falls and injury. In this Special Issue, the work of Geravand et al. (2016) formulates sit-to-stand transfers as an optimal feedback control problem, which optimizes over factors like accuracy, human balance, energy, motion smoothness and human biomechanical constraints. Their approach is evaluated with a robotic assistant and aged persons largely unable to perform sit-to-stand transfers unassisted.

For autonomous robots that serve as task partners, a model of the interaction between the human and robot is both necessary and difficult to define. The Special Issue contribution of Maeda et al. (2016) leverages imitation learning to construct a model of human-robot interaction primitives. Their interaction learning method performs action recognition and enables human-robot movement coordination.

\subsection{Social Robotic Aides}

Social robots' embodiment can engage with the user in the real world - socially, physically and emotionally. Their ability to perceive and produce verbal, nonverbal and affective social behavior allows social robots to become effective educational, therapeutic and cognitive rehabilitation aides, often phrased as socially assistive robots. 
In recent years, many articles report successful robotassisted therapeutic outcomes in children with cognitive impairments. For instance, these robotic agents are equipped with certain verbal (speech), non-verbal (joint and directed attention, emotion and gesture) and interpersonal (turn taking and sharing) functions to study and intervene with children with autism spectrum disorder (ASD) who may lack such social skills. The Special Issue contribution of Chevalier et al. (2016) focuses on the over-reliance on proprioceptive (ability to determine body segment positions and limb movements) information in ASD populations. The authors present a method to create a visual versus proprioceptive sensory reliance profile of a child, and study how facial and body features of a social agent affects each profile group's recognition of the agent's emotion. The authors suggest that such discovery will inform the design of a socially assistive robotic agent (modes of expression and types of embodiment) which will lead to better personalized intervention.

During the foundational workshops, approaches to promote successful collaboration between robotics and clinical researchers were discussed by the organizers and attendees. In this Special Issue, the work of Boccanfuso et al. (2016) points out that using a widely accepted clinical assessment to evaluate the efficacy of the robot-assisted therapeutic protocol is core to this collaboration. This work uses a robot as a confederate for children to practice fun social exchanges before generalizing each task to co-present others. The authors compare improvements observed in a study group receiving the robot-assisted intervention with a control group receiving speech therapy but no robot-assisted intervention. The evaluation of children's social and language skills before and after the session is presented using standard clinical performance measures which show significant improvement in language comprehension and interpersonal and coping skills in children who were in the study group.

Robotic aides' social interaction ability is crucial for users' sustained engagement during therapeutic activities. A robotic exercise coach that provides in-home personalized exercise programs can enhance the quality of life for people who have limited access to other exercise opportunities. The Special Issue contribution of Görer et al. (2016) collects exercise programs provided at local nursing homes to train a robotic coach. This work's contribution is in deploying such a robotic agent for elderly people with visual, hearing and/or cognitive impairments for up to five days to assist their daily exercise routine. A humanoid robot coach successfully engaged the users for the entirety of the interaction using verbal and motion feedback to provide guidance and encouragement to the users. The work of Gross et al. (2016) in this Special Issue aims at developing a socially assistive robot to autonomously coach stroke patients in clinics during their walking and orientation training. A social robotic mobile agent presented in this work is able to navigate itself around the building, accompany and monitor the patient during walking training, and provide feedback and encourage the patient to repeat their exercises regularly. The paper gives an overview of the training scenario and describes the constraints and requirements that arise from the operational environment. The system was carefully tested with clinical staff and then was evaluated with a group of stroke patients.

Cloud-based services provide an effective and efficient approach to enhancing a robot's cognitive abilities. In this Special Issue, the work of Manzi et al. (2016) uses a cloud robotics approach to increase the skills of a robot, endowing the system with abilities for human-robot interaction and environmental sensing. The robot uses text-to-speech and speech recognition services as well as an electronic agenda and web resources to perform several tasks. The system has been tested in a real environment with a couple of elderly users for five days.

\subsection{Wearable Robots: Prostheses and Orthoses}

Recently, wearable robotics technology and related applications have progressed rapidly. Orthotic and prosthetic devices are used to enhance the strength of the wearer, as an assistive device, and for therapy for neurological patients. They could have a great impact on the quality of life of dependent people during daily living activities. Compliant and adaptable impedance-based control approaches applied to exoskeletons/prostheses at the upper/lower limbs have shown great advances in the last decade, due to their ability to provide smooth and transparent physical human-robot interaction. In the Special Issue contribution of Ates et al. (2016), an interactive hand and wrist orthosis for post-stroke rehabilitation provides compliant and adaptable extension assistance at the wrist and fingers. The prototype interfaces with motivational games based on activities of daily living and is integrated with off-the-shelf mobile arm support. Their approach is validated through experiments conducted with patients performing basic activities at home. In the contributed work of Zhao et al. (2016), a combined control Lyapunov function based on quadratic optimization with an impedance controller is proposed, and applied to a self-contained powered transfemoral prosthesis. The results show improvement in tracking performance and energy efficiency of the prostheses control. Experimental work confirms that the controller results in dynamic and stable prosthetic walking with a transfemoral amputee.

When designing a reconfigurable wearable device, gathering information related to kinematic constraints, device characterization and user evaluation is a difficult task. In this Special Issue, Erdogan et al. (2016) propose a powered exoskeleton for the ankle joint that performs this information gathering as well as optimal dimensional synthesis and series-elastic actuation mode control. In their approach, the 
authors present a self-alignment parallel mechanism for the configuration of the prototype kinematics and another parallel mechanism that can support the ground reaction force and torque transferred to the ankle. The resulting prototype has a compact design with low inertia, high fidelity force and impedance control and active back-driveability.

A real-time estimation of the gait phases of human walking is a challenging task, particularly within the context of wearable robots for motion assistance and rehabilitation. The Special Issue contribution of Yan et al. (2016) solves this problem by using a simple sensory apparatus. The proposed system uses a combination of an oscillator-based estimator, a desired gait event detector and a phase-error compensator. Data collected from users' treadmill walking at different speeds and free walking were used to validate the proposed approach.

\section{Conclusions}

From the articles that comprise this Special Issue, and the workshops that served as its base, a number of observations were common across the focus areas of manipulation and mobility assistance, socially assistive robots, and wearable robots. (1) Assistive robots are becoming more and more capable of working more proactively in real human environments. (2) The technologies are becoming more intelligent, with superior perception capabilities-including visual, auditory, haptic and multi-modal—and the ability to communicate information. (3) Reliability has reached a point where human users can physically interact with the robots, and do so safely. (4) The capabilities of assistive robots are diversifying, and are able to meet a larger variety of physical and interactive needs in the daily lives of humans.

The works presented in this Special Issue span theoretical foundations to experimental work, and provide evidence of the necessity and effectiveness of assistive robots. As the field moves forward, critical factors will include the diversity of tasks for which assistance is provided, enhanced functionality for collaboration and communication between the human and robot, personalization to individual users, and the use of common evaluation methods to facilitate collaboration with clinical researchers, to name a few.

\footnotetext{
Acknowledgements The guest editors wish to express their gratitude to Prof. Gaurav Sukhatme, Editor in Chief of Autonomous Robots, the opportunity to publish this Special Issue. We greatly appreciated the responsiveness of Prof. Sukhatme and his staff and their continuous support throughout the submission and review process. The guest editors would like to thank all the keynote speakers and participants in the three successful workshops held at IROS 2014. We also would like to express our gratitude to the many reviewers who kindly provided very thorough and constructive reviews for the papers submitted to this Special Issue.
}

\section{References}

Ates, S., Haarman, C. J., \& Stienen, A. H. (2016). SCRIPT passive orthosis: Design of interactive hand and wrist exoskeleton for rehabilitation at home after stroke. Autonomous Robots, 41(2). doi:10. 1007/s10514-016-9589-6.

Boccanfuso, L., Scarborough, S., Abramson, R. K., Hall, A. V., Wright, H. H., \& OKane, J. M. (2016). A low-cost socially assistive robot and robot-assisted intervention for children with autism spectrum disorder: Field trials and lessons learned. Autonomous Robots, 41(2). doi:10.1007/s10514-016-9554-4.

Chevalier, P., Martin, J. C., Isableu, B., Bazile, C., \& Tapus, A. (2016). Impact of sensory preferences of individuals with autism on the recognition of emotions expressed by two robots, an avatar, and a human. Autonomous Robots, 41(2). doi:10.1007/ s10514-016-9575-z.

Erdogan, A., Celebi, B., Satici, A. C., \& Patoglu, V. (2016). Assist on-ankle: A reconfigurable ankle exoskeleton with series-elastic actuation. Autonomous Robots, 41(2). doi:10.1007/ s10514-016-9551-7.

Geravand, M., Korondi, P. Z., Werner, C., Hauer, K., \& Peer, A. (2016). Human sit-to-stand transfer modeling towards intuitive and biologically-inspired robot assistance. Autonomous Robots, 41(2). doi:10.1007/s10514-016-9553-5.

Görer, B., Salah, A. A., \& Akın, H. L. (2016). An autonomous robotic exercise tutor for elderly people. Autonomous Robots, 41(2). doi:10.1007/s10514-016-9598-5.

Gross, H. M., Scheidig, A., Debes, K., Einhorn, E., Eisenbach, M., Mueller, S., et al. (2016). ROREAS: Robot coach for walking and orientation training in clinical post-stroke rehabilitation-prototype implementation and evaluation in field trials. Autonomous Robots, 41(2). doi:10.1007/s10514-016-9552-6.

Maeda, G. J., Neumann, G., Ewerton, M., Lioutikov, R., Kroemer, O., \& Peters, J. (2016). Probabilistic movement primitives for coordination of multiple human-robot collaborative tasks. Autonomous Robots, 41(2). doi:10.1007/s10514-016-9556-2.

Manzi, A., Fiorini, L., Esposito, R., Bonaccorsi, M., Mannari, I., Dario, P., et al. (2016). Design of a cloud robotic system to support senior citizens: The KuBo experience. Autonomous Robots, 41(2). doi:10. 1007/s10514-016-9569-x.

Schwesinger, D., Shariati, A., Montella, C., \& Spletzer, J. (2016). A smart wheelchair ecosystem for autonomous navigation in urban environments. Autonomous Robots, 41(2). doi:10.1007/ s10514-016-9549-1.

Viswanathan, P., Zambalde, E. P., Foley, G., Graham, J. L., Wang, R. H., Adhikari, B., et al. (2016). Intelligent wheelchair control strategies for older adults with cognitive impairment: User attitudes, needs, and preferences. Autonomous Robots, 41(2). doi:10.1007/ s10514-016-9568-y.

Wachaja, A., Agarwal, P., Zink, M., Adame, M. R., Möller, K., \& Burgard, W. (2016). Navigating blind people with walking impairments using a smart walker. Autonomous Robots, 41(2). doi:10. 1007/s10514-016-9595-8

Yan, T., Parri, A., Garate, V. R., Cempini, M., Ronsse, R., \& Vitiello, N. (2016). An oscillator-based smooth real-time estimate of gait phase for wearable robotics. Autonomous Robots, 41(2). doi:10. 1007/s10514-016-9566-0.

Zhao, H., Horn, J., Reher, J., Paredes, V., \& Ames, A. D. (2016). First steps toward translating robotic walking to prostheses: A nonlinear optimization based control approach. Autonomous Robots, 41(2). doi:10.1007/s10514-016-9565-1. 


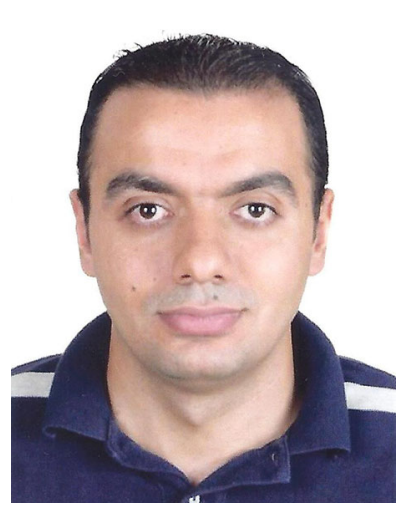

Samer Mohammed received his $\mathrm{Ph} . \mathrm{D}$. degree in computer science from the Laboratory of Computer Science, Robotic and Microelectronic of Montpellier (LIRMM/CNRS), Montpellier. $\mathrm{He}$ is currently an Assistant Professor of computer science with the Laboratory of Image, Signal and Intelligent Systems, University of Paris-Est Créteil, Créteil, France. His current research interests include modeling, identification, and control of robotic systems (wearable robots), artificial intelligence, and decision-support theory. His current research involved the applications for the functional assisting of dependent people. Dr. Mohammed was the recipient of the Fellowship Award from the Japan Society for the Promotion of Science and the French Ministerial Doctoral Research Scholarship Award. He is actively involved in organizing committees of national and international workshops in the automatic and robotic domains.

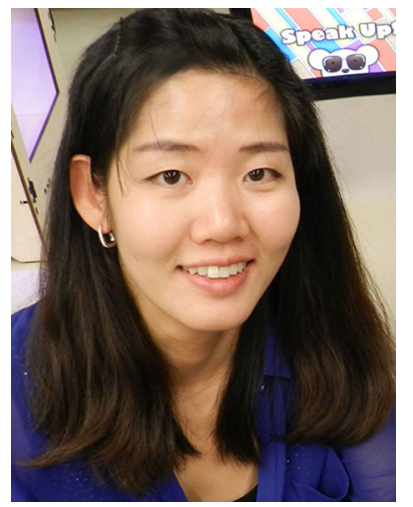

Hae Won Park is a Research Scientist at the Personal Robots Group, MIT Media Lab. She develops robots that can "deep personalize" to their users through long-term interaction, shared experiences and emotions, and interactively learning from each other. She received her $\mathrm{PhD}$ degree at Georgia Tech in 2014 during when she co-founded Zyrobotics, an accessible education robotics company, that is licensing the three technologies developed during her years at Georgia Tech. She received her Master's degree in 2009 from Georgia Tech and Bachelor's degree from POSTECH, Korea in 2006. Before entering graduate school, she was a research scientist at the Cognitive Robotics Center at Korea Institute of Science and Technology (KIST), where she worked on multi-modal object register and recognition for visual SLAM. Her current and past works were featured in MIT Technology Review, NSF Science 360, IEEE Spectrum, TechCrunch, and CNN.

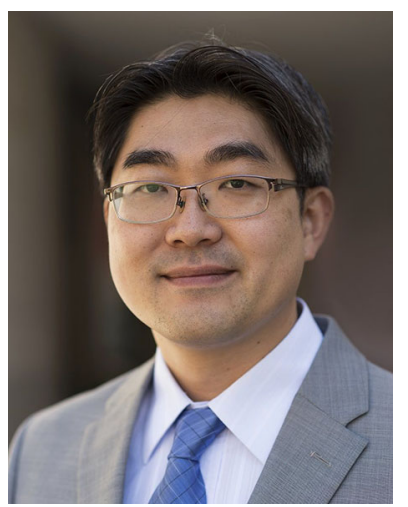

Chung Hyuk Park is currently an assistant professor in the Department of Biomedical Engineering in the School of Engineering and Applied Science (SEAS) at The George Washington University (GW). Prior to joining GW, he was an assistant professor at the New York Institute of Technology and a postdoctoral research fellow at the Georgia Institute of Technology, U.S.A. He received his Ph.D. degree (2012) in Electrical and Computer Engineering from the Georgia Institute of Technology and his M.S. (2002) and B.S. (2000) degrees in Electrical Engineering and Computer Science from Seoul National University, Seoul, Korea. He was also with the LG Electronics Research Center where he received as a team the "LG Electronics Best R\&D Award." He directs the Assistive Robotics and Tele-Medicine laboratory (ART-Med Lab.), with research themes focused on assistive robotics, biomedical systems, machine learning, haptics, computer vision, and multi-modal human-robot interaction.

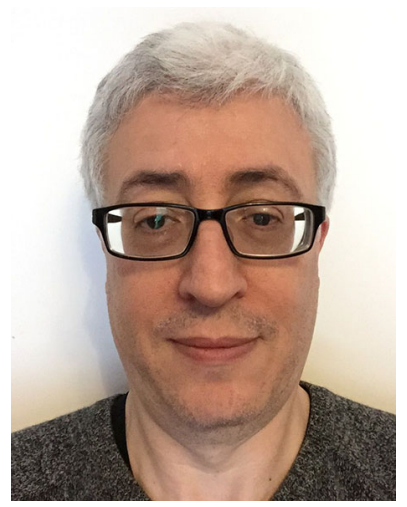

Yacine Amirat received the $\mathrm{Ph} . \mathrm{D}$. degree in computer science and robotics from the University of Pierre et Marie Curie, Paris, France, in 1989, and the Habilitation degree in artificial intelligence and control of complex systems from the University of Paris 12-France, Paris, in 1996. He co-created the Laboratory of Computer Sciences and Robotics, Paris 12 University (newly UPEC), Créteil, France, in 1990 . He is currently the Director of the Laboratory of Image, Signal and Intelligent Systems (LISSI) - UPEC, and the Scientific Director of several research projects. He has authored or co-authored more than 100 papers in scientific journals, books, and conference proceedings. His current research interests include artificial intelligence, soft computing, knowledge processing, and control of complex systems. Application fields are intelligent and robotic systems such as ubiquitous and service robots, wearable robots, and ambient intelligence. Prof. Amirat is a reviewer of many international journals and conférences and a Technical Committee Member, the Session Chair, a Session Organizer, or an Associate Editor of several IEEE-RAS conferences. He is actively collaborating with industrial and academic partners in various European projects.

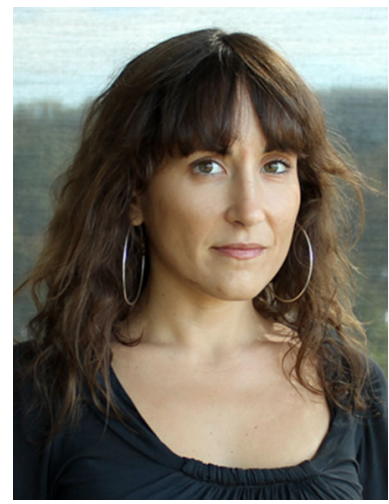

Brenna Argall is an assistant professor in the Departments of Electrical Engineering \& Computer Science, Mechanical Engineering and Physical Medicine \& Rehabilitation at Northwestern University. She also directs a laboratory at the Rehabilitation Institute of Chicago. Her Ph.D. in Robotics (2009) was received from the Robotics Institute at Carnegie Mellon University, as well as her M.S. in Robotics (2006) and B.S. in Mathematics (2002). Prior to joining Northwestern and RIC, she was a postdoctoral fellow (2009-2011) at the École Polytechnique Fédérale de Lausanne (EPFL), and prior to graduate school she held a Computational Biology position at the National Institutes of Health (NIH). Argall's research lies at the intersection of robotics, machine learning and human rehabilitation. The mission of her lab is to advance human ability, by leveraging robotics autonomy. 